\title{
Tracking and flavour tagging selection in the ATLAS High Level Trigger
}

\author{
Milene Calvetti*i \\ University of Pisa and INFN \\ E-mail: milene.calvetti@cern.ch
}

In high-energy physics experiments, track based selection in the online environment is crucial for the efficient real time selection of the rare physics process of interest. This is of particular importance at the Large Hadron Collider (LHC), where the increasingly harsh collision environment is challenging the experiments to improve the performance of their online selection. Principal among these challenges is the increasing number of interactions per bunch crossing, known as pileup. In the ATLAS experiment the challenge has been addressed with multiple strategies. Firstly, specific trigger objects have been improved by building algorithms using detailed tracking and vertexing in specific detector regions to improve background rejection without loosing signal efficiency. Secondly, since 2015 all trigger areas have benefited from a new high performance Inner Detector (ID) software tracking system implemented in the High Level Trigger. Finally, performance will be further enhanced in future by the installation and commissioning of a hardware based Fast TracKer (FTK) throughout 2017.

This presentation will focus on the performance of the ID tracking software as well as looking ahead to projected improvements from FTK. Specific focus will be given to the case of flavour tagging of b-jets, as an example of the implementation of novel algorithms to improve vertexing and light-jet rejection in real time.

EPS-HEP 2017, European Physical Society conference on High Energy Physics

5-12 July 2017

Venice, Italy

* Speaker.

${ }^{\dagger}$ on behalf of the ATLAS Collaboration. 


\section{LHC challenges and the ATLAS trigger}

During 2015 the LHC has been upgraded to bring the center of mass energy to $13 \mathrm{TeV}$ and the bunch crossing separation to $25 \mathrm{~ns}$. The upgrade has also made it possible to reach a record instantaneous luminosity at the price of about 30 interactions per bunch crossing. This challenge has been addressed in ATLAS with multiple strategies: a new pixel detector layer (Insertable B-Layer, IBL), high performance inner detector tracking software (Section 2), improved algorithms on specific physics objects (Section 3) and the installation of the Fast TracKer (FTK) system (Section 4). The ATLAS trigger system consists of a hardware-based first-Level trigger (L1) and a softwarebased High-Level Trigger (HLT) implemented on standard CPUs [1]. The L1 trigger, using input from the calorimeters and from the muon system, reduces the event rate from about $40 \mathrm{MHz}$ to about $100 \mathrm{kHz}$. Events accepted by the L1 trigger are buffered in the Read-Out-System to be processed by the HLT. The HLT receives Region-of-Interest (RoI) information from L1 and, based on the RoI information, requests data from the RoI regions and reconstructs trigger objects. The HLT output rate is about $1 \mathrm{kHz}$.

\section{Inner Detector tracking for trigger algorithms}

The ID tracking software has been redesigned to include IBL information and to make it more flexible so that the HLT tracking can be organised in multiple stages. Track reconstruction uses information from the four-layer pixel detector, the silicon microstrip detector (SCT) and the transition radiation tracker. The improved performance of ID tracking is largely due to the IBL information which results in improved impact parameter resolution. The tracking software has also been modified to be ready to include the tracks provided by the FTK system (Section 4).

The tracking is performed in two distinct steps. The first step, Fast Tracking, consists of triggerspecific pattern recognition algorithms. The second step, Precision Tracking, is largely based on offline reconstruction and uses tracks and clusters provided by the previous step, without repeating the CPU intensive pattern recognition step, and improves their quality. For electrons and muons the ID tracking algorithms are typically executed within the RoIs identified by the L1 trigger. For b-jets and hadronically decayed tau leptons two-stage tracking is performed, with an additional initial fast tracking stage. The first stage of the two-stage tracking is to identify the leading track (for tau leptons) or the primary vertex (for b-jets) within the long but narrow first-stage RoI (Figure 2). The second-stage RoI is then built around the leading track (or primary vertex) identified and both the fast tracking and precision tracking are executed on the second stage RoI. The advantage of the two-stage tracking is that the latency of processing the first and second-stage RoIs is less than for a large single region.

An example of the performances of the two-stage tracking is given in Figure 1, showing the transverse impact parameter $d_{0}$ resolution as a function of the tau lepton transverse momentum $p_{T}$ for tracking at various stages. The two stages of the Fast Track Finder and the Precision Tracking are shown. With the two-stage tracking the $d_{0}$ resolution particularly improves at low $p_{T}$.

\section{3. b-jet trigger}

The goal of b-jet trigger algorithms is to keep as high an efficiency as possible on events 


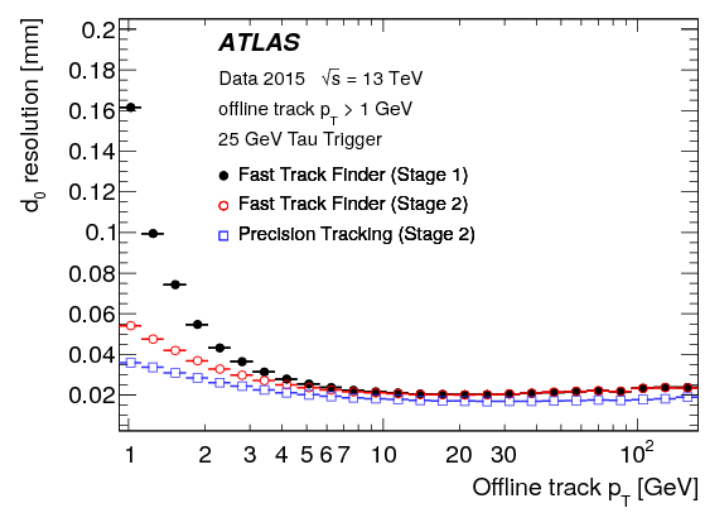

Figure 1: Transverse impact parameter $d_{0}$ resolution as a function of the tau $p_{T}$. The resolution for step 1 of the Fast Track Finder (full circle), the resolution for step 2 of the Fast Track Finder (empty circle) and the resolution for Precision tracking (empty square) are shown [2].

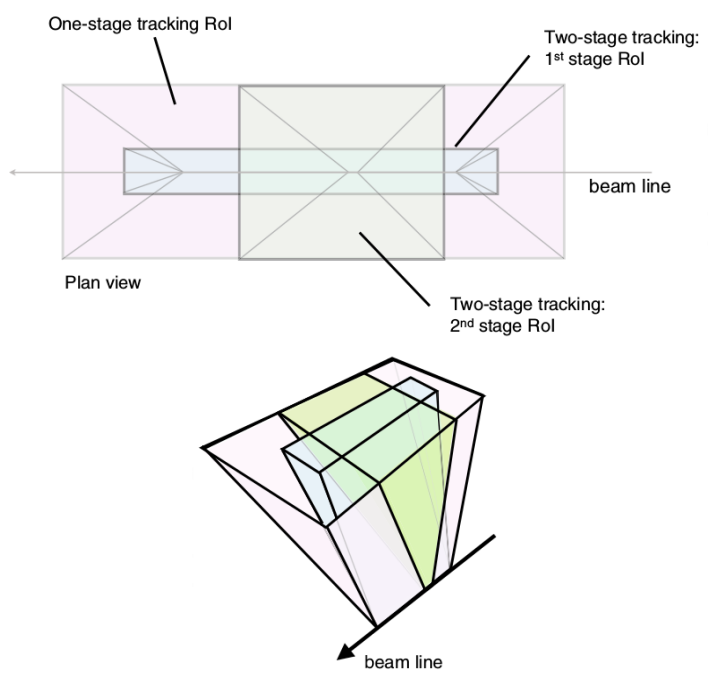

Figure 2: Schematic illustration a RoI used in the single-stage trigger tracking and two RoIs used for the two-stage approach.

containing b-jets while keeping the total rate at an affordable level. Trigger algorithms exploit several b-hadron properties that make it possible to disentangle b-jets from light-jets. The proper decay time of b-hadrons is $1.5 \mathrm{ps}$, therefore, depending on their energy, the hadrons can travel several millimetres in the detector. Consequently, often, a secondary vertex (SV) displaced from the primary vertex can be reconstructed to recognize the decay. Reconstructed tracks associated

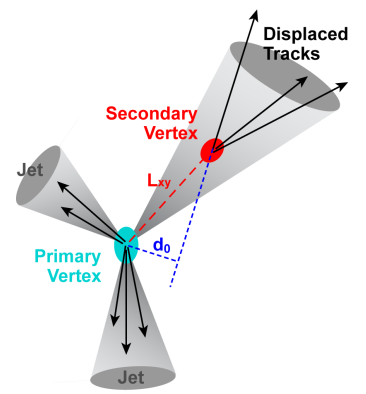

(a)

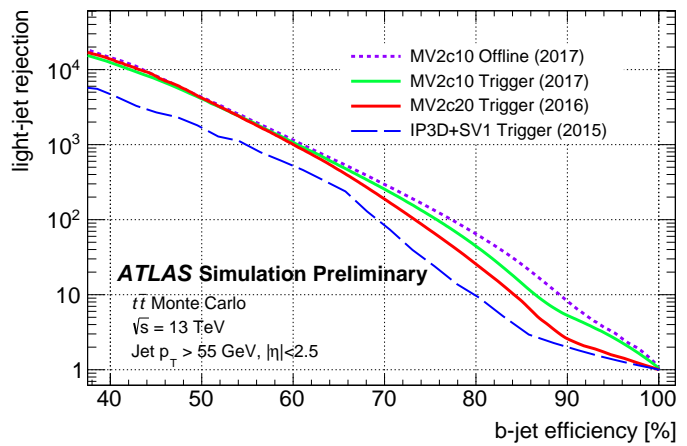

(b)

Figure 3: (a) Schematic illustration of an event with a b-hadron secondary vertex.

(b) Light-jet rejection as a function of b-jet efficiency. Expected performance of b-tagging algorithm for b-jet triggers in 2017 data-taking (green solid line) is compared to b-tagging algorithms used for b-jet triggers in 2016 and 2015 data taking. Performance of b-tagging algorithm for offline jets is shown in purple dotted curve [3].

with this SV have large transverse and longitudinal impact parameters with respect to the primary vertex (Figure 3(a)). In addition, b-hadrons go through hard fragmentation and have a relatively high mass of $(\sim 5 \mathrm{GeV})$. Therefore b-jets, with respect to light-jet, are characterized by higher 
masses, higher multiplicities and a larger fraction of energy carried by tracks.

As b-jet identification heavily relies on track and vertex reconstruction the improved performance of the ID (Section 2) has directly benefited the b-jet trigger. The other major improvement is due to the adaptation of the offline b-tagging algorithms for use in the trigger. The use of the offline MV2 multivariate b-tagging algorithm improved online b-jet identification and increased the level of coherence between the online and offline b-jet identification. The multivariate MV2 algorithm uses inputs from several algorithms exploiting different features of the b-jets:

- The IP3D algorithm exploits the two-dimensional distribution of the longitudinal and transverse impact parameter significance;

- The SV1 exploits properties of the secondary vertex such as the invariant mass of tracks associated to the vertex and the fraction of jet energy associated to the secondary vertex;

- The JetFitter exploits the topological structure of weak b- and c-hadron decays inside the jet.

The performances of several b-tagging algorithms (measured using $t \bar{t}$ Monte Carlo events) are shown in Figure 3(b) in terms of light-jet rejection as a function of b-jet efficiency. The b-jet trigger simulation for 2015 data-taking only uses a combination of the IP3D and SV1 algorithms, while for the trigger simulation of 2016 and 2017 data the MV2 algorithm has been used, which exploits IP3D, SV1 and JetFitter algorithms. The MV2c20 (MV2c10) algorithm is trained assigning bjets as signal and a mixture of $80 \%$ (90\%) light-flavour jets and $20 \%(10 \%)$ c-jets as background. The performance of the offline b-tagging algorithm is shown in Figure 3(b), and is similar to the performance of the online b-jet identification.

\section{Fast TracKer}

The information from the approximately 100 million channels of the tracking detectors is presently exploited in the HLT only for a subset of the events or for limited detector regions due to timing limitations. The Fast Tracker system, that is presently being commissioned, processes full Pixel and SCT data for all events accepted by L1. It will provide, at the L1 trigger rate, the reconstruction of all tracks with $p_{T}>1 \mathrm{GeV}$ with quality very close to offline. Extensive tracking and vertexing is planned to be performed as part of HLT algorithms using FTK tracks and will improve the identification of b-jets, $\tau$ leptons and missing transverse momentum, especially at low $p_{T}$, because the track finding will be run with looser HLT jet thresholds.

To achieve the goal of fast, full event tracking reconstruction FTK is constructed using a combination of dedicated ASIC devices based on associative memories and high performance Field Programmable Gate Arrays. Track reconstruction is divided in various steps, each implemented in dedicated electronic boards. In the following the main track reconstruction steps are described:

- The Pixel and SCT hits are clustered into coarse resolution hits. The clustering reduces the amount of data at the price of a small loss in resolution for Pixel hits, while there is no loss in resolution for SCT. Simulation studies are used to find the best compromise. The data are then divided into $64 \eta \times \phi$ towers and the inputs from the towers are processed in parallel in order to speed up the comparison [4]; 
- The coarse hits are simultaneously compared to millions of pre-stored track patterns (Roads), using only 8 of the 12 Pixel and SCT layers that a track can cross;

- The fine resolution hits are retrieved for all the Roads and a track fit is performed. For each Road the clusters are retrieved at full precision and a track fit is performed using a linear approximation. The tracks with a good fit quality are extrapolated into the remaining 4 layers (IBL and 3 SCT stereo layers) and refitted to improve their quality and to reduce the fraction of fake tracks.

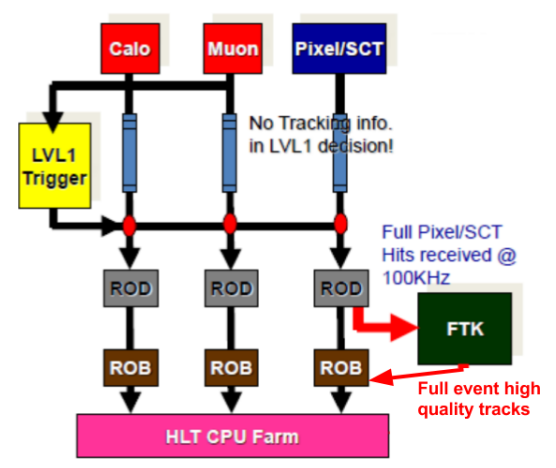

Figure 4: ATLAS trigger diagram including the FTK system.

A schematic diagram of the the ATLAS trigger, including the connections of the FTK system is shown in Figure 4.

\section{Conclusions}

The demanding condition caused by the improved performance of the LHC accelerator have been addressed by the ATLAS experiment with multiple strategies. The trigger algorithms have been improved, a new high performance software tracking system has been implemented in the HLT, the new IBL has been installed and the trigger algorithm have been consequently adapted. A summary of the various updates has been presented in these text. These updates have so far improved the performances of the trigger selection in these more demanding conditions. The performance is expected to further improve when the FTK system is fully commissioned.

\section{References}

[1] ATLAS Collaboration, “The ATLAS Experiment at the CERN Large Hadron Collider," JINST 3 (2008) S08003. doi:10.1088/1748-0221/3/08/S08003

[2] ATLAS Collaboration, "Performance of the ATLAS Trigger System in 2015," Eur. Phys. J. C 77 (2017) no.5, 317 doi:10.1140/epjc/s10052-017-4852-3 [arXiv:1611.09661 [hep-ex]].

[3] ATLAS Collaboration, "Public b-Jet Trigger Plots for Collision Data," https : //twiki.cern.ch/twiki/bin/view/AtlasPublic/BJetTriggerPublicResults.

[4] S. Citraro et al., "Highly Parallelized Pattern Matching Hardware for Fast Tracking at Hadron Colliders," IEEE Trans. Nucl. Sci. 63 (2016) no.2, 1147. doi:10.1109/TNS.2016.2529718 
[5] M. Shochet, L. Tompkins, V. Cavaliere, P. Giannetti, A. Annovi and G. Volpi, "Fast TracKer (FTK) Technical Design Report," CERN-LHCC-2013-007, ATLAS-TDR-021.

[6] A. Annovi et al., "Design of a hardware track finder (Fast TracKer) for the ATLAS trigger," JINST 9 (2014) no.01, C01045. doi:10.1088/1748-0221/9/01/C01045. 